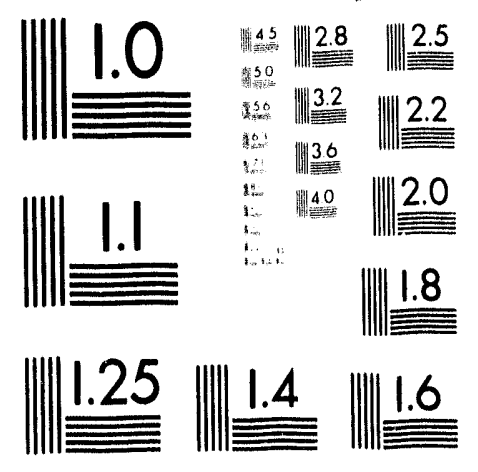



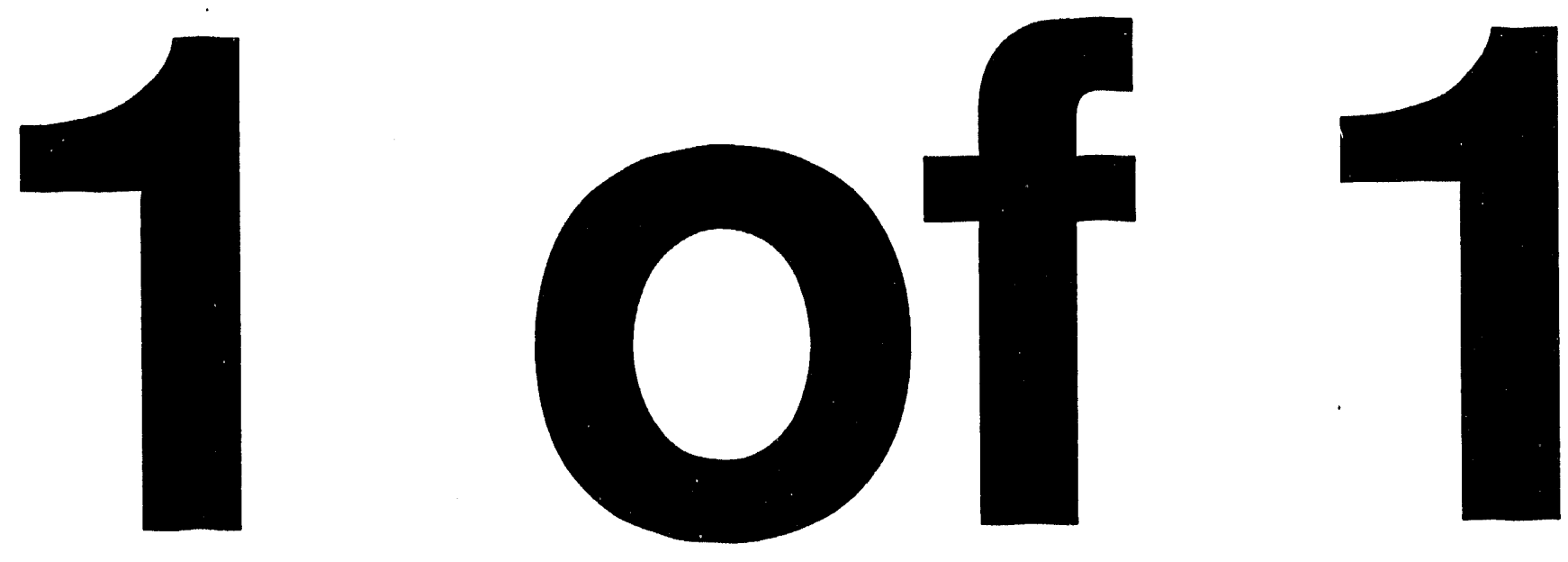

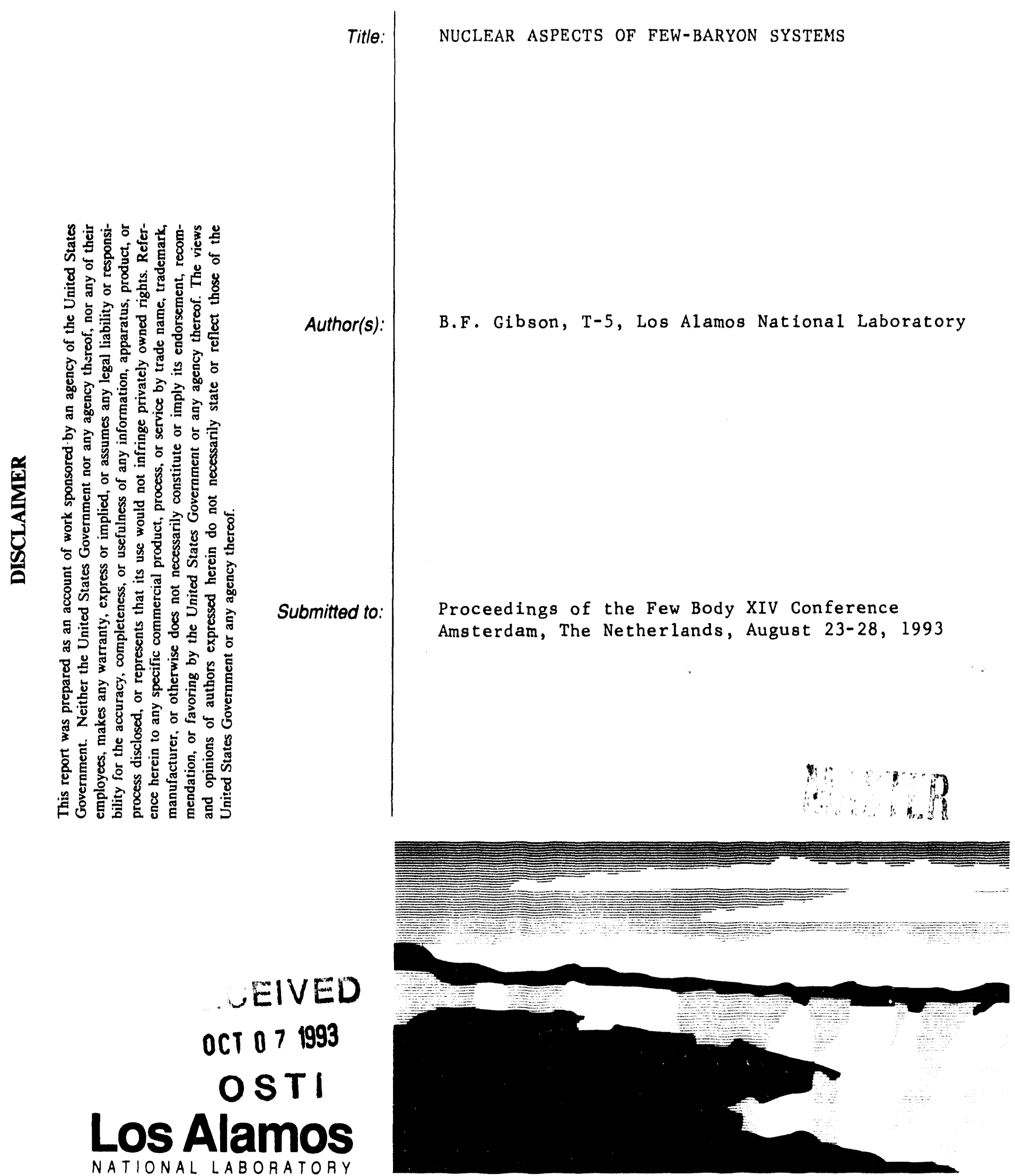

NATIONAL LABORATORY

Los Alamos National Laboratory, an aftirmative action/equal opportunity empldyer, is operated by the University of California for the U.S. Department of Energy under contract W-7405-ENG-36. By acceptance of this article, the publisher recognizes that the U.S. Government retains a nonexclusive, royalty-free license to publish or reproduce the published form of this contribution, or to allow others to do so, for U.S. Government purposes. The Los Alamos National Laboratory requests that the publisher identify this article as work pertormed under the auspices of the U.S. Department of Energy. 


\title{
NUCLEAR ASPECTS OF FEW-BARYON SYSTEMS
}

\author{
B. F. Gibson \\ Theory Division, Los Alamos National Laboratory \\ Los Alamos, NM 87545, USA
}

\begin{abstract}
$\underline{\text { Abstract }}$
Recent progress in understanding the bound state properties of the trinucleons and the alpha particle in terms of a hadron picture of the nucleus is reviewed. The role of three-body forces and meson exchange currents is examined. Novel aspects of few-body hypernuclei as well as unresolved issues in this $S \neq 0$ sector are summarized.
\end{abstract}

I thank the organizers for their invitation to speak at this convocation of European few-body physicists. The topic of few-baryon bound states covers a wide range of intriguing problems, many more than I will be able to touch upon today. Therefore, for a detailed summary of we trinucleon physical observables and model properties, the andience is referred to a review in the proceedings of the Adelaide International Few-Body Conference.[1] Also, a summary of the work by Sasakawa and collaborators will appear shortly in Few-Body Systems.[2] The trinucleon discussion is based upon calculations by a number of people: Friar, Gibson, Payne, ..., Tjon, ..., Glöckle, Witala, ..., Sauer, Stadler, ..., Sasakawa. Ishikawa, ..., Kim, Pickelsimer, Brandenburg, ..., deSwart \& Stoks, .... My brief remarks on ${ }^{4} \mathrm{He}$ are based upon the work of Tjon, Carlson, and Glöckle \& Kamada, .... My comments in this area will be concentrated on recent results and our growing understanding of the physics. The primary emphasis in the talk will be the strangeness $(S=-1 \&-2)$ sector, where hyperons can act as tagged probes of the nucleus. The focus will be on the physics of hypernuclei, where a number of novel features are to be found and a number of unresolved issues remain.

\section{The Strangeness-Zero Sector}

Before summarizing the status of our understanding of the low energy physical observables for the conventional $(S=0) A=3 \& 4$ bound states, a brief glimpse of the history of calculational developments is in order. Tjon [3] gave us the early calculations of ${ }^{3} \mathrm{H} /{ }^{3} \mathrm{He}$ properties using a realistic potential. The analysis of those impulse approximation charge form factors is still valid today: triton underbinding implies radii that are too large, and the diffraction minima occur at too high a momentum transfer in nonrelativistic Hamiltonian model calculations. Afnan [4] produced the initial ${ }^{3} \mathrm{~S}_{1} \cdot{ }^{3} \mathrm{D}_{1},{ }^{1} \mathrm{~S}_{0}$ (5-channel) calculation for the exisiting realistic force models, using the Adhikari-Sloan expansion; 
the Ernst-Shakin-Thaler expansion has since been used [5] for contemporary potential model calculations. Recently, the W-matrix expansion of Sandhas, Haberzettl, .. [6] has been shown to give reliable results for a low-rank, small-basis solution and holds promise for speedy nd $\rightarrow$ nnp breakup calculations. Finally the coordinate-space approach of Kok and Schellingerhout [ 7$]$ appears to be a computationally efficient means of attacking the 3-body and 4-body problem.

\section{Traditional Model}

The traditional approach to nuclear physics can be defined in terms of the constraints placed upon the complexity of the model assumptions:

- Nuclei consist only of nucleons - other degrees of freedom are suppressed.

- N'ıcleons move slowly within the nucleus - nonrelativistic dynamics prevails.

- Nucleons interact primarily via pairwise (two-body) forces.

This is an enormous simplification of the physics, but it accounts amazingly well for much of the experimental data. Nonetheless, our calculational ability has achieved the precision required to see differences between traditional model predictions and experiment, and much research during the past decade has been focussed upon extensions: meson exchange currents (MEC), three-body forces (3BF), $N N-N \Delta$ coupling, relativistic dynamics, quarkgluon substructure, .... I will concentrate on results from the traditional model for binding energies (and implicitly other low energy observables) and address only briefly the MEC, $3 \mathrm{BF}$, and relativity aspects of extensions.

Before turning to specific systems, let us take a quick look at the nonrelativistic assumption. The $A=3 \& 4$ bound states have charge radii of the size of $1.5-1.8 \mathrm{fm}$, the same order as the pion compton wave length $\left(1 / \mathrm{m}_{\pi}\right)$. The uncertainty principle permits one to estimate $\Delta \mathrm{p}$ from the size of the system: $\langle p\rangle \approx 120 \mathrm{MeV} / \mathrm{c}$. Also, from the triton kinetic energy of $\approx 40 \mathrm{MeV}$, one can estimate $\left\langle p^{2}\right\rangle^{1 / 2} \approx 160 \mathrm{MeV} / \mathrm{c}$. Thus, the average momentum of the system is of the order of $m_{\pi}$, and

$$
\left(\frac{u}{c}\right)^{2} \approx\left(\frac{\langle p\rangle / M}{c}\right)^{2} \approx\left(\frac{m_{\pi}}{M}\right)^{2} \approx 2 \%
$$

Caveat: This is an average. It should reflect accurately relativistic corrections to the low energy observables, which are averages over the nuclear volume. However, processes involving high momentum wave function components can suffer much larger relativistic effects.

\section{Status of the Trinucleons}

It has now been reasonably well established that the low energy observables "scale" with the trinucleon binding energy.[8, 9] A summary of results for charge radii, wave function probabilities, magnetic moments, Coulomb energy, asymptotic normalization cr tants, and $\mathrm{Nd}$ scattering lengths can be found in Ref. [1]. Because of this scaling pl. 
Benchmark results exist from a number of groups [8. 9. 10,11, 12, 13, 14, 15, 16] for a variety of realistic potentials $[17,18,19,20,21]$. In this context realistic implies

- strong spin-isospin dependence $\left(V_{n n} \neq V_{n p}\right)$,

- strong tensor force (OPEP is essential, providing up to $3 / 4$ of the potential energy in ${ }^{3} \mathrm{H}$ and ${ }^{4} \mathrm{He}$ ),

- strong short range repulsion (the probability of $N N$ overlap at such separations should be small),

in addition to a reasonable fit to the $N N$ scattering data. Somewhat surprisingly, the explicitly momentum-dependent models such as Paris and Nij78 are more "pulsive in the negative-parity partial waves in a triton binding energy calculation than are the RSC and AV14 models.[22]

The latest development of consequence is a set of local potential models by the $\mathrm{Ni}$ jmegen group with a $x^{2} / \mathrm{f} \approx 1$ for the scattering observables. [23] These charge dependent potential models (with $V_{n n} \neq V_{n p}^{s} \neq V_{n p}^{\prime}$ ) have now been used to estimate the triton binding energy to be $\mathrm{B}\left({ }^{3} \mathrm{H}\right)=7.62 \pm .01 \mathrm{MeV} .[24]$ Thus, a local potential model of the $N N$ interaction that fits the $N N$ observables as well as any phase shift analysis leads to underbinding of the triton by about $0.85 \mathrm{MeV}$.

Previously such underbinding of the triton by local potential models had led investigators to raise the question of the role of 3BF. 225] Various groups demonstrated that adding a two-pion-exchange $(\pi \pi)$ three-body force [26. 27] to the Hamiltonian led to overbinding of the triton when the $\pi N N$ vertex cutoff of $5.8 \mathrm{~m}_{\pi}$, as recommended by the authors of Ref. [26], was used.[28, 29, 30, 31] For example, for the combined RSC/TM model one obtains $\mathrm{B}\left({ }^{3} \mathrm{H}\right) \simeq 8.9 \mathrm{MeV}$ for $\mathrm{j} \leq 2$ (or $9.1 \mathrm{MeV}$ for $\left.\mathrm{j} \leq 4\right)$. Recently Coon and Peña completed the TM 3BF by deriving the $\pi \rho$ and $\rho \rho$ terms.[32] Now, Stadler et al.[31] have calculated the ${ }^{3} \mathrm{H}$ binding energy for the full TM 3BF. Just as the $\rho$ cancels part of the $\pi$ contribution to the $N N$ force, the $\pi \rho$ term cancels part of the $\pi \pi$ term in the $N N N$ force. The result for the $\mathrm{RSC} / \mathrm{TM}$ model is that $8.9 \mathrm{MeV} \rightarrow 8.4 \mathrm{MeV}$. (Similarly, for the Paris/TM combination one finds $9.1 \rightarrow 8.5$ and for the Nij78/TM model one finds $9.3 \rightarrow$ 8.7.) Apparently, a nonvelativistic: Hamiltonian composed of a local $N N$ potential plus a comparable $3 B F$ can yield approximatley the correct value for $\mathrm{B}\left({ }^{3} \mathrm{H}\right)$.

Where do the Bonn potentials fit in this picture? Let me remind everyone that the Bonn q-space potentials (as approximations to the full Bonn energy-dependent interaction) are not local. That is, they do not depend solely upon the difference $|\vec{p}-\vec{p}|$. Nonlocal interactions have an added degree of flexibility, which may well be partially mimicked in local Hamiltonians by a 3BF. Recall the theorem by Polyzon and Glöckle [33] that relates the three-body potential and on-shell equivalent two-body interactions. Paraphrasing: If there exist two-body Hamiltonians $H_{12}\left(=T_{1}+T_{2}+V_{12}\right)$ and $\vec{H}_{12}$ which have the same binding energies and scattering matrices, are complete, and are related by a unitary transform, then there is a three-body interaction $W$ which relates the three-body Hamiltonians $\left(T_{1}+T_{2}+T_{3}+V_{12}+V_{13}+V_{23}\right)$ given by $H$ and $\bar{H}^{\prime}=\bar{H}+W$ such that they have the same binding energies and scattering matrices. In other words, it is quite conceivable that if 
a local potential phus 3BF describes the physies of the trimeleons, then there is also a nonlocal potential plus different 3BF (perhaps identically zero) that will also describe the physies. The Bonn potentials may already incorporate certain 3BF effects in their nonlocal nature. Using the T.M 3BF with the Bonn NN potential is likely donble connting at some level, as the result for the Bonn-Q/TM calculation [31] indicates. However, it also worth noting that the difference between the charge-dependent Bom-B potential triton, $\mathrm{B}\left({ }^{3} \mathrm{H}\right)=7.92 \mathrm{MeV},[16]$ and the nonlocal Nijmegen charge-dependent potential (Nijm I) result, $\mathrm{B}\left({ }^{3} \mathrm{H}\right)=7.72 \mathrm{MeV}[24]$ is only some $200 \mathrm{keV}$. Are the model differences so great? The Graz group is examining the electromagnetic response of the $N$ N system in an effort to pin down the proper off-shell behavior. [34]

For the experimentalists, let me reiterate that three-body forces is a "game" for theorists. On the of her hand, we now have molerstood much about the "rules of the game."

The Alpha Particle

Tjon first established that the $A=3 \mathrm{~d}+$ mucleon system binding energies are strongly correlated.[35] This is in part due to the fact that the ${ }^{3} \mathrm{~S}_{1} \cdot{ }^{3} \mathrm{D}_{1}$ interaction is so dominant. That is, $\left\langle V_{n p}^{t}\right\rangle \gg\left\langle V_{n p}^{*}\right\rangle \Rightarrow$ the physics of all the s-shell nuclei must be similar.

The Gr:IC results of Carlson [36] confirm that underbinding for ${ }^{3} \mathrm{H}(\simeq 7.7 \mathrm{MeV}$ for the AV14 rodel) and for ${ }^{4} \mathrm{He}(\simeq 24.9 \mathrm{MeV})$ follows the Tjon line. Adding a phenomenological $3 \mathrm{BF}$ to fit the triton implies a corresponding fit for the $\alpha$ particle.

Integral equation solutions by Glöckle and Kamada for realistic force models have just appeared.[3i] In addition to binding energies for the AV14 model (7.68 MeV and 24.62 Mel), they quote results for several other potentials for $\mathrm{j} \leq 3 N N$ partial waves. One should note Kamada's talk [38] later in the week as well as Fonseca's review [39] of the $A=4$ system.

Meson Exchange C'urrents

The deviation of the ${ }^{3} \mathrm{H} /{ }^{3} \mathrm{He}$ magnetic moments from the Schmidt limits provided an early indication of the need to include subuncleon degrees of freedom when nucleons are embedded within the nuclear medium. In one langnage these effects are due to MEC. Isovector corrections [40] are estimated to be of the order of $15 \%$ and are reasonably model independent. [41] The isoscalar MEC contributions to the magnetic moments are expected to be small, of the order of relativisitc corrections. Indeed, the isoscalar magnetic moment combination for ${ }^{3} \mathrm{H}$ and ${ }^{3} \mathrm{He}$ appoars to be roasonably described by impulse approximation. [41]

What is known in the case of MEC corrections to the charge operator? It is tempting to invoke them to obtain a fit to the charge form factor data. An ad hoc addition to the impulse approximation form factor of a term which vanishes at $q^{2}=0$ and is negative in the region of the diffraction minimum and beyond will certainly improve the fit to the clata. Such a procedure is far from unicque. In the case of the deuteron, an isoscalar object, we have seen [42, 43] that the difference between relativistic results and the nonrelativistic impulse approximation is small for a significant range of momentum transfer. This is in contrast with the large effect that one sees when relativistic current corrections are 
included as a perturbation. In his Les Houches lectures. Tjon dissected the relativistic calculation for OPE. [4t] He demonstarted that inclurling the relativistic corrections to the current operator alone can be extremely misleading. The effect is large, but incorrect. Specifically, the MEC correction is ancelled by a corresponding dynamical correction to the ${ }^{2} \mathrm{H}$ wave function the nucleon (an be offshell. When modeling isoscalar (relativistic) corrections to the ${ }^{3} \mathrm{H} /{ }^{3} \mathrm{He}$ form factors, the practitioner should first check the model results for the ${ }^{2} \mathrm{H}$ form factor.

\section{Hypermuclei}

A hypernucleus is a baryon system comprised of conventional (nonstrange) nucleons and one or more strange hyperons ( $Y=1, \Sigma$, or $\equiv$ ). The presence of the strangeness degree of freedom (Havor) adds a new dimension to our evolving picture of muclear physics. Because the $S=-1$ hyperon masses differ markedly from those of the mentron and proton, $S U(3)$ symmetry is clearly broken. How it is broken is a question of fundamental importance to our unclerstanding of the baryon-baryon interaction. The seareh for the $S \neq 0$ dibaryon states directly bears upon this. Certainly the investigation of strangeness in few-baryon systems will play a significant role in our understanding the strong force.

The physics of hypernuclei has proven to be both novel and puzzling, stretching our intuition and analysis (apability beyond that developed during the more than half century that we have explored conventional nuclear physics. The hypernuclear sector of hadronic physics is not just a simple extension of zero-strangeness phenomena. The discoveries have been new; the physics is different. We have observed such novel aspects as:

- obvious charge symmetry breaking in the ${ }_{1}^{4} \mathrm{He}-{ }_{1}^{4} \mathrm{H}$ isodoublet. $[45,46]$

- anomalously small binding of ${ }_{\Lambda}^{5} \mathrm{He},[45,47,48,49,50]$

- surprisingly narrow structure in the ${ }^{4} \mathrm{He}\left(\mathrm{K}^{-}, \pi^{ \pm}\right)$reactions, a possible ${ }_{i}^{4} \mathrm{He}$ hypernucleus. $[51,52,53]$

These phenomena stand out clearly without the need for sophisticated theoretical analysis of the data. Furthermore, the following aspects are also interesting:

- The "H" dibaryon ( $S=-2$ uuddss object) is a unique prediction of the QCD Bag model.[54]

- Theoretical calculations imply a significant role for 3BF effects in the hypertriton ${ }_{i}^{3} \mathrm{H} .[55,56]$

- A spin inversion between the $A=4$ ground states and excited states wonld explain unobserved $\gamma$ rays in heavier hypernuclei. [57, 58, 59]

- Confirmation of a claim for the observation of ${ }_{.1}^{\text {He would argue }}$ strongly against the existence of a deeply bonnd "H". $[60)]$ 
I hypernuclear plypsices has bern established as an interesting subject, one which deserves concentrated effort to comprehend. The prospect of exploring $\Sigma$ hypermuclei and the more exotic $S=-2$ systems makes the strange physies sector that much more attractive.

The available data on the fow-body 1 hypernuclei come primarily from emmlsion experiments [45] binding energics and weak decay properties. We shall discuss only the binding energies today, for wo have seen in the $S=0$ sector that binding energy determines the low energy olservables. In the study of hypermuclei, it is customary to cunote the $A$-separation encrgies

$$
B_{1}(A A)=B(. A)-B(A-1) \text {. }
$$

The accepted values for the s-shell systems are quoted in Table 1 along with the measured $\gamma$-ray deexcitation energies for the two species with particle-stable excited states. The $A=6$ entry is $B_{\Lambda \Lambda}=B\left({ }_{A \Lambda}^{6} \mathrm{He}\right)-B\left({ }^{4} \mathrm{He}\right) \cdot[60]$ I would point out that Don Davis of University College London is a great source of information about the cmulsion data.[61. 62]

Table 1. Hyperunclear . 1-separation energies and excitation energies in $\mathrm{MeV}^{\circ}$

\begin{tabular}{ccc}
\hline hypernucleus & $\mathrm{B}_{1}$ & $\mathrm{E}_{9}$ \\
\hline${ }_{1}^{3} \mathrm{H}$ & $0.13 \pm .05$ & \\
${ }_{1}^{4} \mathrm{H}$ & $2.04 \pm .04$ & $1.04 \pm .04$ \\
${ }_{1}^{5} \mathrm{He}$ & $2.39 \pm .03$ & $1.15 \pm .04$ \\
${ }_{1}^{5} \mathrm{He}$ & $3.10 \pm .02$ & \\
${ }_{1}^{4} \mathrm{He}$ & 10.6 & \\
\hline
\end{tabular}

\section{The YV Interaction}

Several novel features of the $Y V$ interaction come into play in hyperunclear physies. The $\perp(T=0)$ and $N(T=1 / 2)$ cannot exchange a $\pi(T=1)$, so that there is no dominant OPE tensor force in $\Lambda N$ scattering. Shorter range properties of the baryonbaryon interaction play a more important role than in $N$ s scattering. The longest range components are due to the exchange of two pions or one kaon. The shorter range $\mathrm{K}$ exchange potential does admit a tensor-force component, but it is largely cancelled by that from $K^{*}$-exchange. Thus, tensor-force effects in the.$N$ interaction are experted to be smaller than those in the $N N$ interaction. $[63,64,65,66,67,68]$

On the other hand, $M N-\Sigma V$ compling effects are expected to be much more important in the hypermuclear sector than are $. N N-N \Delta$ compling effects in the nonstrange sector. The

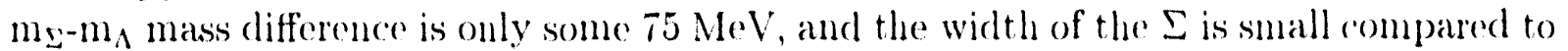
that of the $\Delta$. Formally eliminating the $\Sigma$ channel from the problem leads to an energy dependent $\Lambda N$ effective interaction and to $\triangle N N$ three-body forces. Both of these effects have been the subject of recent research in the nonstrange sector.[14]

The experimental lata for $\Lambda N$ and $\Sigma . V$ scattering consist of some 600 events in the low energy range (momenta of less than $300 \mathrm{MeV} / \mathrm{c}$ ) [69, 70, 71] and another 250 events 
in the momentum range 300-1500 MeV/ [i2]. A phase shift analysis is not a practical possibility. The low energy data fail to adeduately define even the relative sizes of the dominant s-wave spin-triplet and spin-singlet seattering lengths. [09] The limited cross section measurements have been grouped with the abundant $N N$ data in a combined analysis to constrain the YN OBE potential models. The Nijmegen group has devoloped several OBE models by simlutaneonsly fitting the $N . V$ and $Y N$ data and employing $S U(3)$ constraints among the coupling constants. In addition, the Jiilich group has now constructed $Y N$ potential models with a different character. In particular, the assumption about the F/D ratio (scalar singlet and scalar octet coupling) differs, which affects the relative strengths in the $\Lambda N$ and $\Sigma N$ channels.

The scattering lengths and effective ranges for five of these models are collected in Table 2. The scatter among the parameters gives an indication of how poorly the potentials are constrained by the data. The scattering lengths are clearly negative, so that there is no bound hyperdeuteron. However, one can not determine whether the interaction in the spin-singlet state or the spin-triplet state is the stronger. A cusp is expected in the region of the threshold for $\Sigma$ production. although neither the position nor the magnitude of the peak in this energy region is determined by the data; the cross sections in this anergy region exhibit uncertainties of a factor of $2-4$. The strong $1.2-5 . V$ coupling combined with the sizeable $\Sigma^{-}-\Sigma^{+}$mass difference and the charge dependence of the $\Sigma N$ ()BE force implies a measureable charge symmetry breaking in the $Y N$ interaction, which is clourly seen in the $A=4$ isodoublet.

Table 2. The scattering lengths and effective ranges in $\mathrm{fm}$ for the $Y N$ potential models listed

\begin{tabular}{lc|cccc}
\hline Model & Ref. & $\mathrm{a}^{\mathrm{s}}$ & $\mathrm{r}_{0}^{\mathrm{s}}$ & $\mathrm{a}^{\mathrm{t}}$ & $\mathrm{r}_{0}^{\mathrm{t}}$ \\
\hline Nijmegen D & {$[63]$} & -1.90 & $3 . \overline{7}^{2}$ & -1.96 & 3.24 \\
Nijmegen F & {$[64]$} & -2.29 & 3.17 & -1.88 & 3.36 \\
Nijmegen SC & {$[65]$} & -2.78 & 2.88 & -1.41 & 3.11 \\
Jiilich A & {$[68]$} & -1.56 & 1.43 & -1.59 & 3.16 \\
Jiilich $\tilde{A}$ & {$[68]$} & -2.04 & 0.64 & -1.33 & 3.91 \\
\hline
\end{tabular}

\section{$\Lambda$ Hypernuclei}

The question remains open as to whether we can successfully calenlate the properties of the few-body $\triangle$ hypermuclei utilizing realistic $Y N$ and $N N$ interactions. Becanse neither the $\Lambda N$ nor $\Sigma_{N}$ forces possess sufficient strength to support a bound state, the ground state of the $\triangle N N$ system (the hypertriton) plays the role of the denteron. The spin and parity of ${ }_{\Lambda}^{3} \mathrm{H}$ were determined to be $J^{*}=\frac{1}{2}^{+}$from an analysis of the angular distribution of pions emitted during its weak mesonic decay. The small .1-separation energy (130 \pm 50 $\mathrm{keV}$ ) leads one to expect a loosely bound system - essentially a $\Lambda$ rlinging temonsly to a deuteron. As such, one expects the hypertriton to be sonsitive to the long range properties of the $Y N$ interaction. Because $\Lambda N-\Sigma N$ coupling plays a large role in that 
interaction, one is also led to sperenhate whether ${ }_{1}^{3} \mathrm{H}$ is bound only beranse of the . I.Y. 3BF that results when the I diamel is eliminated from the formalism.

Because of the weak binding and lack of definitive $). V$ scattering data. many theorists have studied ${ }_{3}^{3} \mathrm{H}$ using separable potentials fitted to the approximate . I. L low energy parameters. (For a partial list. see Ref. [55].) The essential physies ran be slummarized

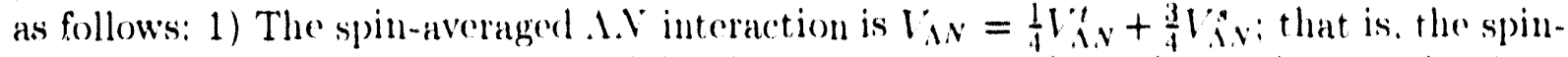
singlet interaction dominates. 2) The dispersive energy dependence that results from embedding the A.V-S.V compled-channel potential in a three-body system is repulsive, as is that from the $N N-N J$ interaction in the triton. 3) The true $3 B F$ due to the coupling of $\Sigma N N$ states to $I N N$ states is attractive. 4) The $Y N$ tensor force interacts with the $N N$ tensor force in a complex manner. which depends upon the relative sign of the ${ }^{3} \mathrm{~S}_{1}$ ${ }^{3} \mathrm{D}_{1}$ mixing parameters. This analysis has been confirmed by the reent calculation of Miyagawa and Glöckle [56] using the Jiilich A model in combination with varions realistic $N N$ potentials. Surprisingly, the model ${ }_{1}^{3} \mathrm{H}$ was found to be mubound. This is surprising because the low encrgy scattering parameters for it in Table 2 wonld imply that the $\triangle N V$ system should bind: thus. one must ronclucle that the short range properties of the realistic potential play an mexpected role in this weakly bound sistem. The binding of the hypertriton is clearly a balancing act. A $4 \%$ enhancement in the strength of lifi. would yield a bound state [j6]. but such a change in the spin-singlet interaction would destroy the model A fit to the Y'X data.

The ${ }_{1}^{4} \mathrm{He}-\mathrm{H}$ isodoublet provides an even stronger test of our modeling of the $Y . \mathrm{V}$ interaction. The quality of the calculations for the (ground and excited) states of the $A=4$ system should approach that demonstrated for the a particle. Even greater precision should be obtained for the charge-symmetry-breaking (CSB) difference

$$
\Delta B_{A}=B_{\Lambda}\left({ }_{1}^{4} \mathrm{He}\right)-B_{A}\left({ }_{1}^{4} H\right) \text {. }
$$

The nominal $\triangle B_{1} \simeq 350 \mathrm{keV}$ is much larger than the $\simeq 100 \mathrm{keV}$ CSB effect seen in the ${ }^{3} \mathrm{He}-{ }^{3} \mathrm{H}$ binding energy difference after correcting for the $p p$ Conlomb energy in ${ }^{3} \mathrm{He}$. A key question is whether the CSB can be understood in terms of the free. $\mathrm{V}$ interaction. The question is not trivial. Consider a simple separable-potential model fitted to the $1 p$ and In scattering parameters of Nijmegen D listed in Table 3. A folding model approximation for the $A$-nucleus potential

$$
V_{i A}(k)=\int d^{3} p \rho_{A}(\vec{p}) I_{i N}(p, k)
$$

which provides effective two-body dyuamias in a Schrödinger equation solution, yields a value of $\Delta B_{1}=0.21 \rightarrow 0.2+\mathrm{MeV}$ when a core compression of $0 \rightarrow 5 \%$ is used. In contrast, an exact four-body (alculation for the identical potentials vields a value of $\Delta B_{1}=0.43$ MeV - almost twice the folding model result. [46] Few-body dynamics does matter.

The existence of particle-stable excited states for the $A=4$ system provides a further strong constraint upon our models of the $Y^{*} N$ interaction. Fitting both $t+n^{+}$and $1^{+}$states within the same model is not a trivial exercise. The spin structum is more complex than one might naively expect; the ${ }_{1}^{4} \mathrm{H}$ states are not simply $\left[1 \otimes^{3} \mathrm{H}\right]^{[\mathrm{J}]}$ configurations. Furthermore, $. N-\Sigma N$ coupling plays an important role because of the composite 
Table 3. The charge-dependent scattering lengths and effective runges in fun for Nijmegen D

\begin{tabular}{c|cccc}
\hline channel & $\mathrm{H}^{4}$ & $\mathrm{r}_{0}^{\mathrm{s}}$ & $\mathrm{n}^{\prime}$ & $\mathrm{r}_{0}^{\prime}$ \\
\hline $\mathrm{Np}$ & $-1.7 \mathrm{1}$ & 3.78 & -2.06 & 3.18 \\
$\mathrm{~N} n$ & -2.03 & 3.66 & -1.84 & 3.32 \\
\hline
\end{tabular}

nature of the trinucleon core states. A simple example follows from the Stepien-Rudza and Wycech [73] separable potential approximation to Nijmegen D. The spin-avernged potential combination that determines the $0^{+}$state is $\frac{1}{2} V_{i_{N}}+\frac{1}{2} V_{\mathrm{iN}}$, whereas for the $1^{+}$ state it is $\frac{1}{6} V_{i v}+\frac{5}{6} V_{.}$. If explicit $\Lambda . V-\Sigma N$ coupling is neglected, and the $A N$ effective interactions are constructed from the scattering lengths and effective ranges, then one finds in all exact four-body calculation that

$$
\mathrm{B}\left(0^{+}\right) \simeq 10.7 \mathrm{MeV} \quad \text { and } \mathrm{B}\left(1^{+}\right) \simeq 11.7 \mathrm{M} \cdot \mathrm{V}:
$$

the ground state and excited states are inverted, the $1^{+}$being more bound. However. if one takes into acount the composite nature of the $A=3$ core states and suppresses the $T=3 / 2$ trinucleon excited states that comple to the $\Xi$ but lie $10 \mathrm{~s}$ of MoV higher in energy, then one fiuds

$$
\mathrm{B}\left(0^{+}\right) \simeq 9.6 \mathrm{MeV} \text { and } \mathrm{B}\left(1^{+}\right)=8.2 \mathrm{MeV} \text {. }
$$

which places the states in proper order.[59] This simplified schematic-model calculation emphasizes the importance of $\triangle N-\Sigma N$ coupling. That has been born out in variational calculations using Nijmegen $\mathrm{SC}$ for the $Y N$ interaction. For the $0^{+}$state, where the singlet and triplet interactions are of cqual importance, one finds $B_{1}\left(0^{+}\right) \simeq 1.5 \mathrm{MeV}$, in clualitative agreement with the data. However. the $1^{+}$state is mbound. (74) The spin-triplet interaction which dominates the $1^{+}$system derives much of its attraction in Nijmegen SC from the.$\leftrightarrow$ S transition term l'x. in the compled channel potential

$$
\left[\begin{array}{ll}
\text { IXN } & \text { UXN } \\
\text { IXN } & \text { nN }
\end{array}\right]
$$

which is less effective "in medium" than in free space, just as is tensor coupling in the $N N$ interaction.

The anomalously small binding of ${ }_{1}^{5} \mathrm{He}$ remains an enigma. In the baryon picture, the four nucleons and $A$ can coexist in $1 s$ states. Simple model calculations based upon AN potentials paraneterized to account for the low energy scattering data. as well as the binding energies of the $A=3 \&+$ systems, overbind ${ }_{1}^{5} \mathrm{He}$ by $2-3 \mathrm{MeV} .[48,49,50]$ Explanations of the model overbinding have been proposed in terms of tensor forces that bind ${ }^{3} \mathrm{H}$ and ${ }^{4}$ He less than do central forces, [47] and in terms of $. N-\Sigma N$ coupling that is weakened when the nucleon is part of a composiite core. [75] The variational calculations by Carlson again fail to bind, because of the strength in the.$\leftrightarrow \mathrm{H}$ transition potential. 


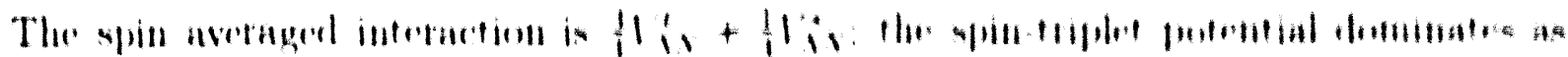

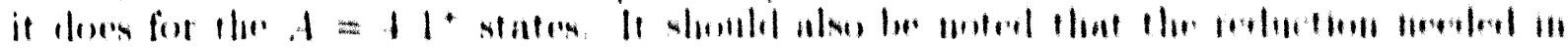

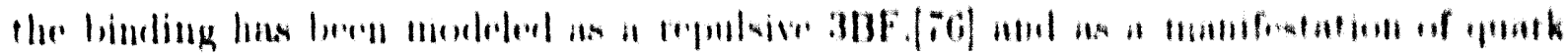
ifferets.(iT)

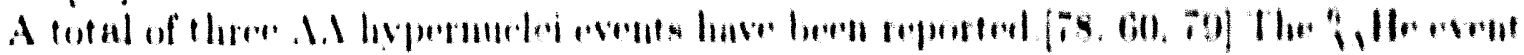

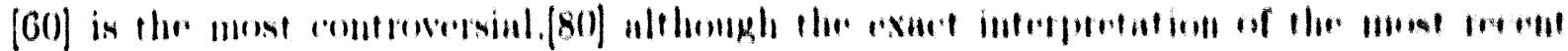

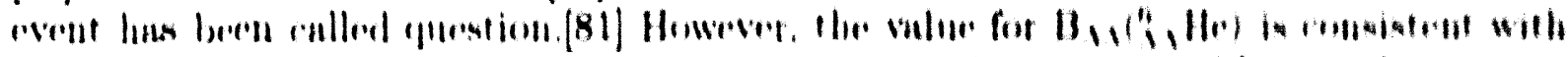

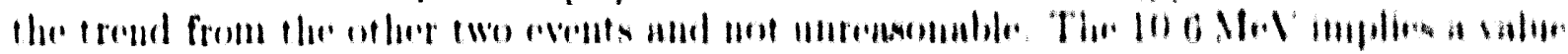

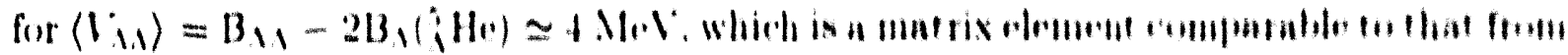

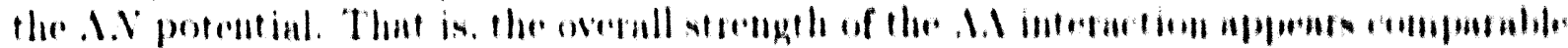

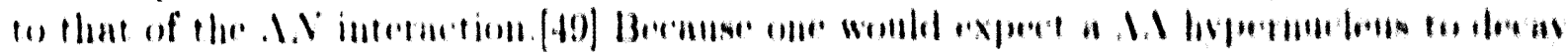

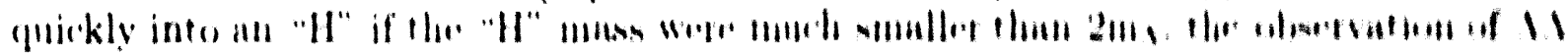

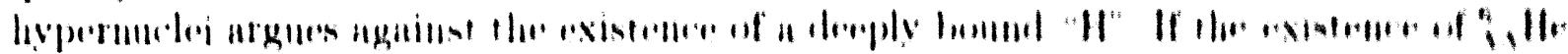

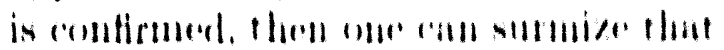

$$
111122111,-11) .11 \cdot 1
$$

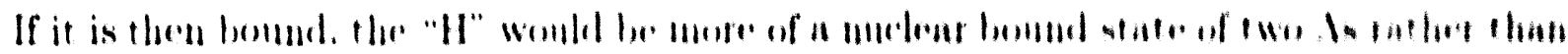
a deoply boulud undelss exotice.

EHypermunloi

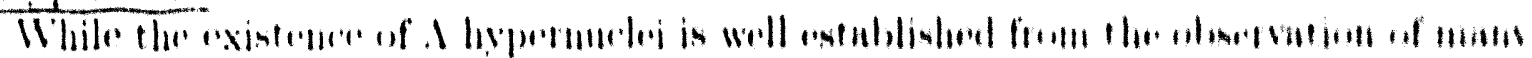

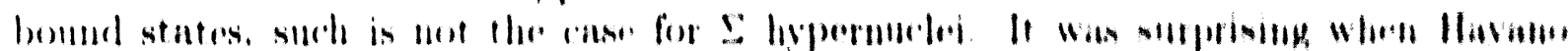

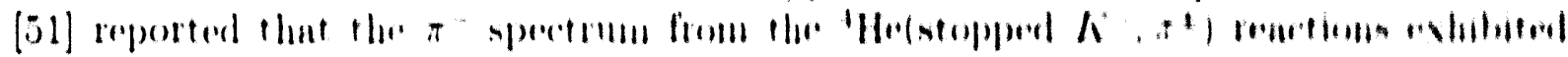

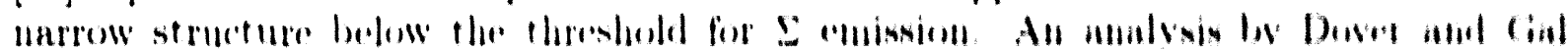

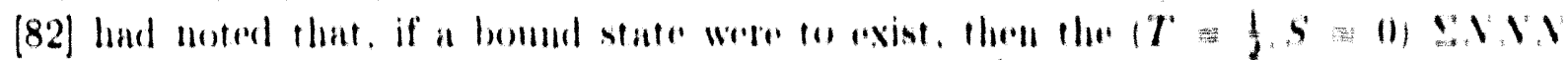

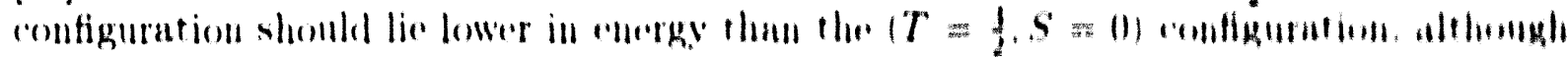

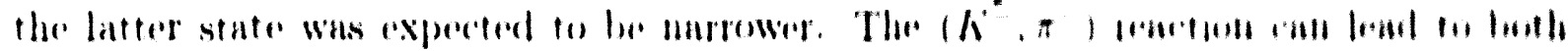

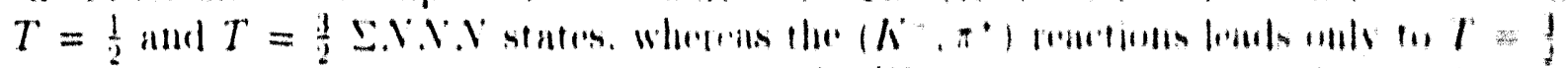

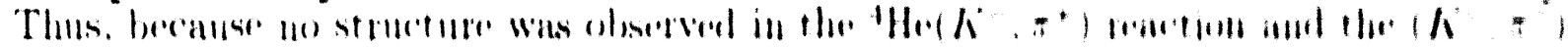

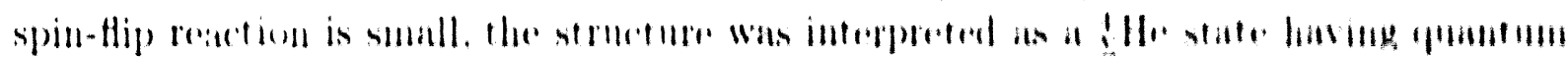

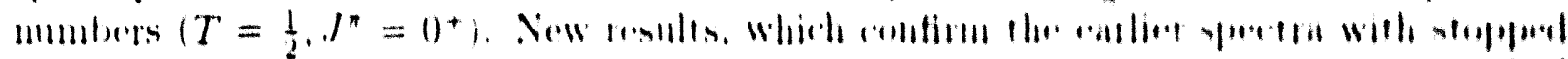

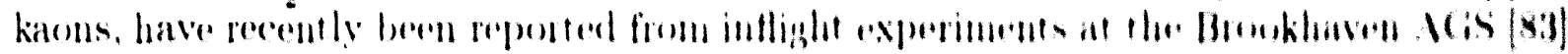

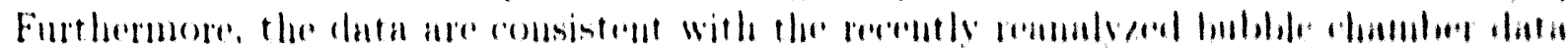

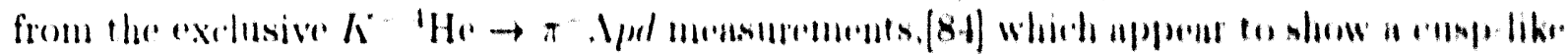

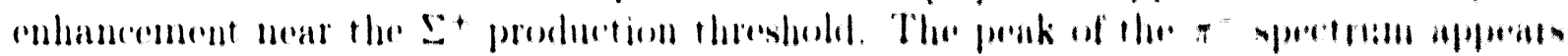

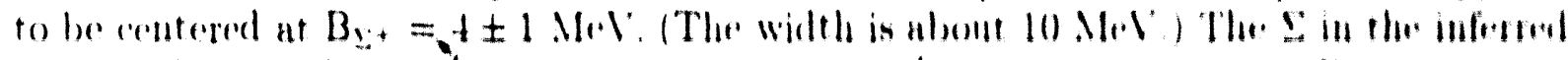

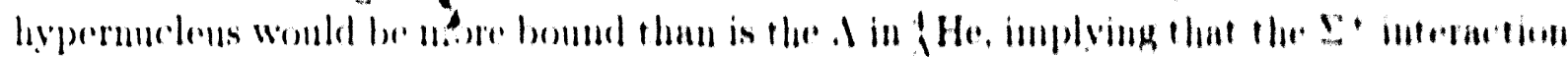

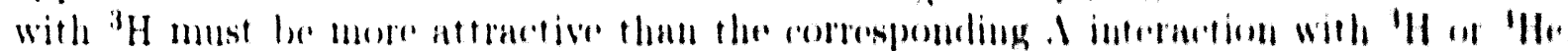

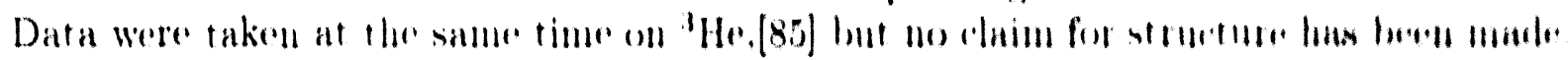

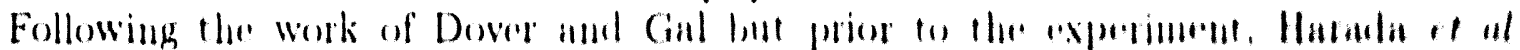

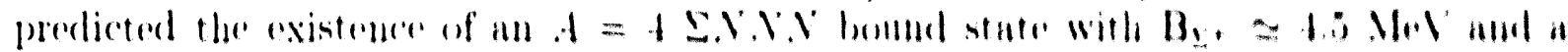

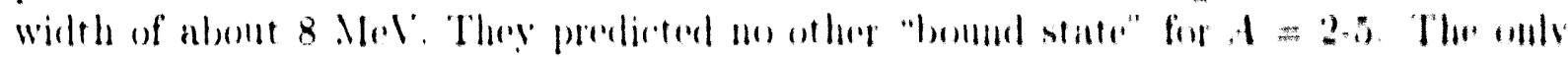

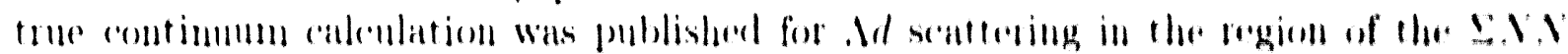


Plireshold.[80] Surpriningly. it was fomml that structure in the elastic scattering cross

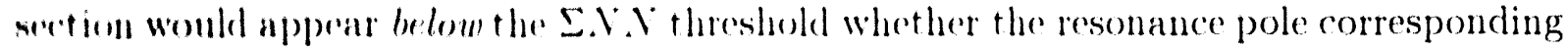

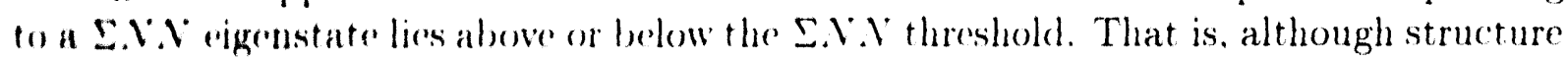
in the cross section helow threshold does imply the existence of a resonance, it does not puarantere that the pole lies below threshold (that one has a "bound state").

\section{Acknowledgement}

The work of the anthor was performed under the auspices of the U. S. Department of Energy. Ho gratefully arkinowledges a Research Award for Senior Scientists by the Alexander von Humboldt Stiftung which marle possible his stay with the I. K. P. Theorie gromp at the Forsehungszentrmm Jiilich, where this manuscript was prepared.

\section{References}

(1) Gibson, B. F.: Nucl. Plyss. A543. 10 (1992).

[2] Sasakawa. T. Ishikawa. S.: Few-Body Sys. (to appear)

[3] Tjon, J. A. et. al:: Pliys. Rev. Lett. 25. 540 (1970).

(4) Afuan. I. R.. Read. J. M.: Phys. Rev. C 8. 1294 (1973); 12, 293 (1975).

[5] Koike, Y.. ef al. Physs. Rev. C 35, 396 (1987); Haidenbauer, J., Koike, Y': Phýs. Rev. C' 34.118i (1986).

(6) Bartnik. E. A.. et al.: Phỵs. Rev. C' 36, 1678 (1987):

[i] Sehellingerhout. $\therefore$. W: see contribution to this volume.

[8] Friar. I. L.. et al.: Physs. Lett. B 161, 241 (1985).

[9] Ishikawa, S., Sasakawa, T.: Few-Body Sys. 1, 3 (1086).

[10] Payne. G. L.. et al.: Plyys. Rev. C 22, 823 (1980); Chen. C. R., et al.: Phys. Rev. C 31. 2266 (1985); Friar, J. L., et al.: Phys. Rev. C 37.2869 (1988).

(11) Hajduk. C'.. Sauer, P. L.: Nucl. Phys. A369, 361 (1981).

[12] Bömellurg, A.: Phỵs. Rev. C' 28, 403 (1983).

[13] Ishikawa. S., et al.: Physs. Rev. Lett. 53, 1877 (1984).

[14] Sauer. P. L': Prog. in Part. Nucl. Phys. 16. 35 (1986).

(15) Brandenburg. R. A. ct al.: Physs. Rev. C 37, 124j (1988). 
[16] Witala, H., et al.: Phys. Rev. C' 43, 1619 (1991).

[17] Reid, R. V., Jr.: Ann. Phys. (NY) 50, 411 (1968); Day, B.: Phys. Rev. C 24, 1203 (1984) - labeled RSC'.

[18] Nagels, M. M., et al.: Pliys. Rev. D 17, 768 (1978) - labeled Nij78.

[19] Lacombe, M., et al.: Phys. Rev. C 21, 861 (1980) - labeled Paris.

[20] Wiiringa, R. B., et al.: Phys. Rev. C 29, 1207 (1984) - labeled AV14

[21] Machleidt, R., et al.: Phys. Rep. 149, 1 (1987) - labeled Bonn-R and Bonn-Q;

Machleidt, R.: Adv. Nucl. Physics, 19, 189 (1989) - labeled Bonn-B.

[22] Payne, G. L., Gibson, B. F.: Few-Body Sys. 14. 117 (1993).

[23] Stoks, V. G. J., Klomp, R. A. M., deSwart, J. J.: private communication.

[24] Friar, J. L., et al.: Phys. Lett. B 311, 4 (1993).

[25] Gibson, B. F., McKellar, B. H. J.: Few-Body Sys. 3, 143 (1987).

[26] Coon, S. A., et al.: Nucl. Phys. A317, 242 (1979); Coon, S. A., Glöckle, W.:

Phys. Rev. C 23, 1990 (1981) - labeled TM.

[27] Coelho, H. T., et al.: Phys. Rev. C 28, 1812 (1983); 31, 646 (1985) - labeled BR.

[28] Chen, C. R., et al.: Phys. Rev. C 33, 1740 (1986).

[29] Ishikawa, S., Sasakawa, T.: Few-Body Sys. 1, 143 (1986).

[30] Bömelburg, A.: Phys. Rev. C 34, 14 (1986).

[31] Stadler, A., Adam, J., Henning, H., Sauer, P. U.: "Triton calculations with $\pi$ - and $\rho$-exchange three-nucleon forces" (submitted to Phys. Rev. C).

[32] Coon, S. A., Peña, M. T.: Phys. Rev. C (to appear).

[33] Polyzou, W. N., Glöckle, W.: Few-Body Sys. 9, 97 (1990).

[34] Pauschenwein, J.: Few-Body Sys. Suppl. 6, 195 (1993).

[35] Tjon, J. A.: Phys. Lett. B 56, 217 (1975).

[36] Carlson, J. A.: private communication.

[37] Glöckle, W., Kamada, H.: Phys. Rev. Lett. 71, 971 (1993).

[38] Kamada, H.: see contribution to this volume.

[39] Fonseca, A. C.: see contribution to this volume. 
[40] Riska, D. O., Brown, G. E.: Phys. Lett. B 38, 193 (1972).

[41] Friar, J. L. et al:: Phys. Rev. C 37, 2852 (1988).

[42] Zuilhof. M. J., Tjon, J. A.: Phys. Lett. B 84, 31 (1979); Phys. Rev. C' 22, 2369 (1980).

[43] Arnold, R. C., et al:: Phys. Rev. C 21, 1426 (1980).

[44] Tjon, J. A.: in: Hadronic Physics with Multi-GeV Electrons, p. 89. New York: Nova Science Publ. (1985); Nucl. Phys. A463, 157c (1987).

[45] Juric, M. et al: Nucl. Phys. B52, 1 (1973).

[46] Gibson, B. F., Lehman, D. R.: Nucl. Phys. A329, 308 (1979).

[47] Bodmer, A.: Phys. Rev. 141, 1387 (1986).

[48] Herndon, R. C., Tang, Y. C.: Phys. Rev. 153, 1091 (1967); 159, 853 (1967); 165, 1093 (1968); Dalitz, R. H. et al.: Nucl. Phys. B47, 109 (1972).

[49] Gibson, B. F. et al:: Phys. Rev. C 6, 741 (1972).

[50] Gal, A.: Adv. Nucl. Physics 8, 1 (1975).

[51] Hayano, R. S. et al: Nuovo Cimento 102A, 437 (1989): Phys. Lett. B 231. 355 (1989).

[52] Harada, T. et al:: Nucl. Phys. A507, 715 (1990).

[53] Hayano, R. S.: Nucl. Phys. A527, 477 (1991).

[54] Jaffe, R. L.: Phys. Rev. Lett. 38, 195 (1977).

[55] Afnan, I.R., Gilsson, B. F.: Phys. Rev. C 40, Ri (1989); 41, 2787 (1990).

[56] Miyagawa, K., Glöckle, W.: Phys. Rev. C (to appear).

[57] Bamberger, A. et al.: Nucl. Phys. B60, 1 (1973).

[58] Bejidian, M. et al: Phys. Lett. B 83, 252 (1979).

[59] Gibson, B. F., Lelman, D. R.: Pliys, Rev. C 37079 (1988).

[60] Prowse, A.: Phys. Rev. Lett. 17, 782 (1966).

[61] Davis, D. H.: in: Proceedings of the LAMPF Workshop on $(\pi, K)$ Physics, A. I. P. Conf. Proc, 224, p. 38. New York: Amer. Inst. Phys. (1991).

[62] Davis, D. H., Sacton, J.: in: High Energy Physics, Vol. II, p. 365. New York: Academic Press (1967). 
[63] Nagels, M. M. et al.: Phys. Rev. D 15, 2547 (1977).

[64] Nagels, M. M. et al.: Phys. Rev. D 20, 1633 (1979).

[65] Massen. P. M. M. et al.: Phys. Rev. C 40, 2226 (1989).

[66] Holzenkamp, B. et al.: Nucl. Phys. A500, 485 (1989).

[67] Holinde, K.: Nuc. Phys. A547, 255c (1992).

[68] Reuber, A. G. et al:: Czech. J. Phys. 42, 1115 (1992).

[69] Alexander, G. et al:: Phys. Rev. Lett. 13, 484 (1964); Phys. Rev. 173, 1452 (1968).

[70] Englemann, R. et al.: Phys. Lett. 21, 587 (1066).

[71] Sechi-Zorn, B. et al.: Phys. Rev. 175, 1735 (1968).

[72] Kadyk, J. A. et al.: Nucl. Phys. 27, 13 (1971).

[73] Stepien-Rudza, W., Wycech, S.: Nucl. Phys. A 362, 349 (1981).

[74] Carlson, J. A.: in: Proceedings of the LAMPF Workshop on $\left(\pi, K^{*}\right)$ Physics, A.I.P. Conf. Proc. 224, p. 198. New York: Amer. Inst. Phys. (1991).

[75] Gibson, B. F. et al:: in: Few Particle Problems in Nuclear Interactions, p. 188. Amsterdam: North Holland (1972).

[76] Bodmer. A. R., and Usmani, Q. N.: Nucl. Phys. A477 621 (1988); Phys. Rev. C 31 , 1400 (1985); Bodmer, A. R. et al.: Phys. Rev. C 29, 684 (1884).

[77] Hungerford, E. V., Biedenharn, L. C.: Phys, Lett. B 42, 232 (1984).

[78] Danysz, M. et al:: Phys. Rev. Lett. 11, 29 (1963); Nucl. Phys. 49, 121 (1963).

[79] Aoki, S. et al.: Prog. Theo. Phys. 85, 1287 (1901).

[80] Dalitz, R. H. et al:: Proc. Roy. Soc. London A426, 1 (1989).

[81] Dover, C. B. et al.: Phys, Rev. C' 44, 1905 (1901).

[82] Dover, C. B., Gal. A.: Phys. Lett. B 110443 (1982).

[83] Hayano, R. S.: Nucl. Phys. A547, 151c (1992).

[84] Dalitz, R. H. et al.: Phys. Lett. B 236, 76 (1990).

[85] Hungerford, E. V.: Nucl. Phys. A547, 157c (1992).

[86] Afnan, I. R., Gibson, B. F.: Plyys. Rev. C' 47, 1000 (1993). 

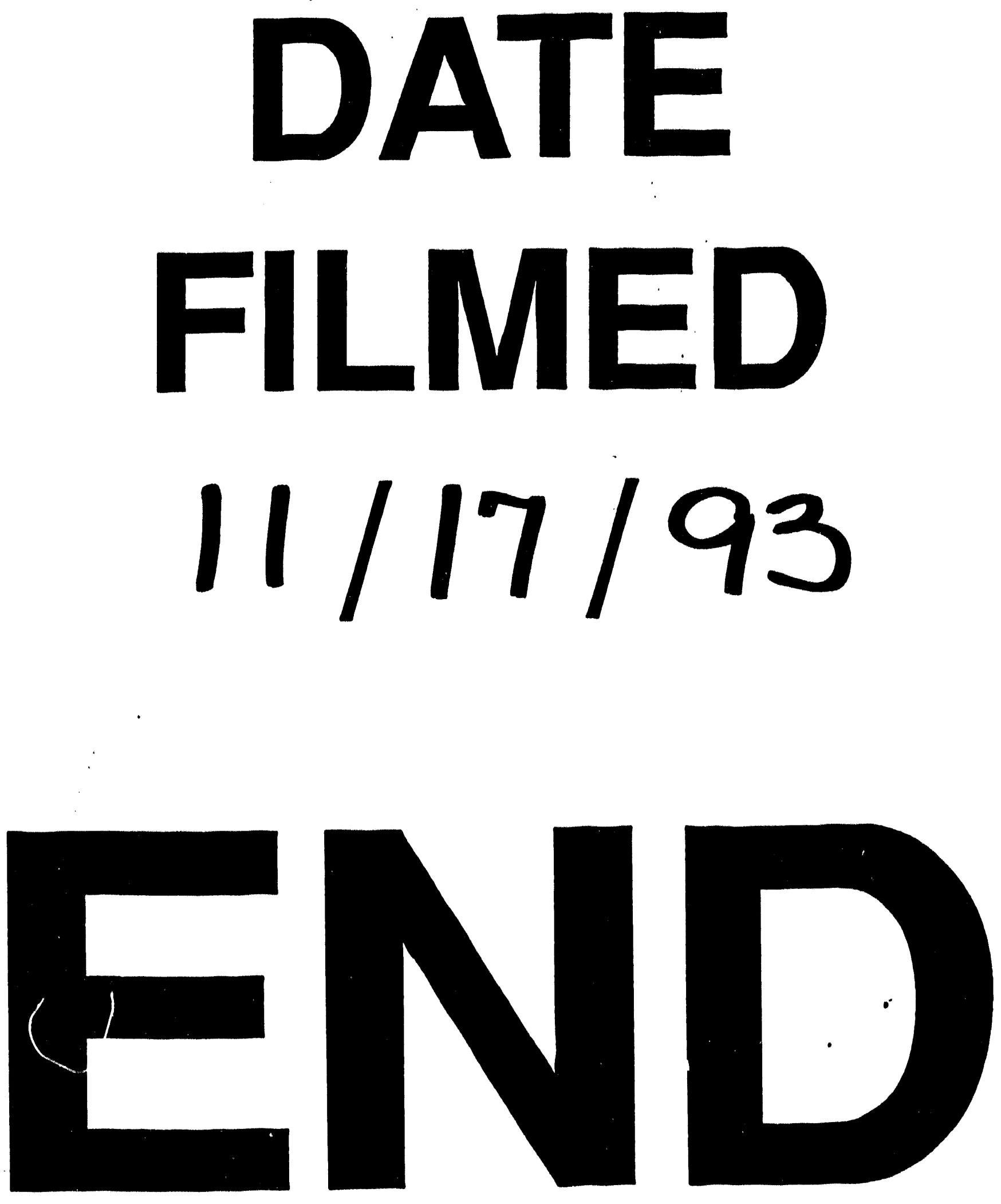
